University of Nebraska - Lincoln

DigitalCommons@University of Nebraska - Lincoln

Faculty Publications from the Department of Electrical \& Computer Engineering, Department Electrical and Computer Engineering

2008

\title{
A Quantitative Study of Mobility Impact for Real- Time Services on a Wi-Fi Multi-hop Network
}

\author{
Ting Zhou \\ University of Nebraska-Lincoln \\ Hamid Sharif \\ University of Nebraska-Lincoln, hsharif@unl.edu \\ Michael Hempel \\ University of Nebraska-Lincoln, mhempel2@unl.edu \\ Puttipong Mahasukhon \\ University of Nebraska-Lincoln \\ Wei Wang \\ University of Nebraska-Lincoln \\ See next page for additional authors
}

Follow this and additional works at: https://digitalcommons.unl.edu/electricalengineeringfacpub

Part of the Electrical and Computer Engineering Commons

Zhou, Ting; Sharif, Hamid; Hempel, Michael; Mahasukhon, Puttipong; Wang, Wei; and Ci, Song, "A Quantitative Study of Mobility Impact for Real- Time Services on a Wi-Fi Multi-hop Network" (2008). Faculty Publications from the Department of Electrical and Computer Engineering. 157. https://digitalcommons.unl.edu/electricalengineeringfacpub/157

This Article is brought to you for free and open access by the Electrical \& Computer Engineering, Department of at DigitalCommons@University of Nebraska - Lincoln. It has been accepted for inclusion in Faculty Publications from the Department of Electrical and Computer Engineering by an authorized administrator of DigitalCommons@University of Nebraska - Lincoln. 


\section{Authors}

Ting Zhou, Hamid Sharif, Michael Hempel, Puttipong Mahasukhon, Wei Wang, and Song Ci 


\title{
A Quantitative Study of Mobility Impact for Real- Time Services on a Wi-Fi Multi-hop Network
}

\author{
Ting Zhou, Hamid Sharif, Michael Hempel, Puttipong Mahasukhon, Wei Wang, Song Ci \\ Computer and Electronics Engineering Department \\ University of Nebraska-Lincoln \\ NE 68182-0572
}

\{tzhou, hsharif, mhempel, pmahasukhon, wwang, sci2\}@ unl.edu

\begin{abstract}
In this paper, we analyze the performance of mobile real time services in a large-scale IEEE 802.11 multi-hop network. We present our field measurements with discussion of performance bottlenecks for VoIP services under mobile scenarios. We utilized our test bed which is located in Nebraska and supported by the University of Nebraska-Lincoln (UNL), US Federal Railroads Administrations (FRA), and major US railroad companies. Our UNL-FRA test bed consists of 8 outdoor access point towers, which are deployed along 3.5 mile of BNSF railroad. Passive measurement approaches are taken to ensure the integrity of collected data and multi-layer stream based packet analyzer has been implemented to provide a global view of the entire performance of the monitored network from the physical layer to the application layer. Based on our analysis of collected data, we conclude that in a typical outdoor 802.11 environment similar to our UNL-FRA test bed, uncertain handoff latencies and lack of Quality of Service guarantee are main performance bottlenecks for real-time applications. Furthermore, we discuss the enhancement strategies to support mobility in high-speed railway networks. We believe our work is one of the initial efforts in wireless mobile network field measurement in terms of scale, methodology, and analysis.
\end{abstract}

Keywords- Passive measurement, performance study, mobility, multi-hop, 802.11

\section{INTRODUCTION}

Today 802.11 [1] based networks have become a de facto standard and been used as a wide-area wireless technology due to unlicensed radio spectrum, low-cost equipment and end-toend IP support, though it suffers from having smaller cells than EDGE and WCDMA systems [2], which with the same transmit power and antenna gain, its range is just one-third that of the latter two.

To be considered as a candidate for supporting mobility, 802.11 is required to guarantee quality of services, or at least provide seamless service to mobile users as well as to stationary ones. However, limited reports have been published discussing the behaviors of large scale outdoor 802.11 multihop networks under mobility. Therefore, this is one of the motivations for our work.

A passive measurement approach is chosen to measure the entire network's traffic as it does not introduce additional traffic overhead and not interact with the monitored network. However, there are many challenges of applying it in largescale mobile railway networks. These include:

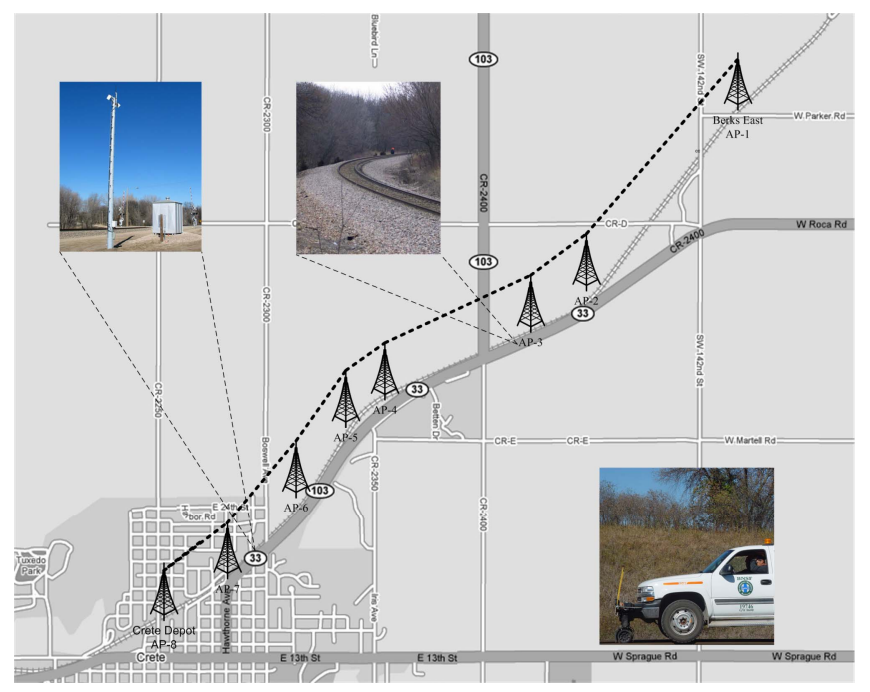

Figure 1. The layout of the access points (AP) in the UNL-FRA testbed, located in Crete, NE, consists of 8 outdoor access point (AP) towers. A HyRail vehicle, which is offered by BNSF, is employed to drive on the track, as well as on the road close to the track to collect data.

(a) Propagation latency due to large-scale outdoor environment;

(b) Monitoring the entire target network where multiple monitors have to be utilized. Additionally, the local time needs to be synchronized frequently to minimize the impact of the system clock inconsistencies;

(c) Due to propagation differences, the status of a transmitted packet in monitor traces may be different with its actual status and need to be corrected.

To address the above challenges, a unique measurement approach has been proposed to merge multiple monitor's traces to estimate the propagation latency with microsecond accuracy. A set of finite state machines (FSM) has been designed to restore the real status of captured packets.

Also a multiple-layer stream-based packet analyzer has been implemented to provide a global view of the entire performance of the target network: from wireless physical layer performance, such as wireless frequency and received power, to the application performance can be measured. For example, the whole lifetime of a real time VoIP stream can be traced and its service quality can be estimated.

The data was collected from a representative railway testbed (UNL-FRA), which is located in Crete, NE, including 8 
outdoor access points and has a total length of 3.5 miles. This particular segment is chosen because of its representative nature, which features several turns, but also straight segments of track. It is in parts surrounded by dense foliage, while in others is open and unobstructed. The test bed includes 8 outdoor access points, which each includes 4 MAC interfaces: two $802.11 \mathrm{a}$ interfaces for wireless backhaul, one $802.11 \mathrm{~b} / \mathrm{g}$ interface for client access, and one local management interface (using the IEEE 802.3 protocol). To avoid a performance bottleneck, an internal wire speed switch is used to interconnect them. The detailed description for this test bed is reported in [3] and the layout of this test bed is shown in Fig.1.

The remainder of this paper is organized as follows: in Section 2 the important aspects of the IEEE 802.11 MAC protocol and prior work in WLAN measurement are reviewed. Data collection and measurement methodology are described in Section 3. The measurement results on the mobility performance are demonstrated in Section 4. Finally based on our measurement results, we discuss the MAC layer optimization strategies for enhancing mobility performance.

\section{BACKGROUND AND RELATED WORK}

When a station moves along the track in our FRA-UNL WLAN testbed and gradually out of the service coverage area of its current AP, the received signal strength from its current AP will decrease while the bit error rate (BER) increases, which results in more MAC frame retransmissions. When the number of retransmissions becomes greater than a given threshold or a given number of beacon frames are continuously missing, then a STA may look for a more suitable AP by scanning channels.

There are two ways to discover a suitable AP: active discovery and passive discovery. For the former, the wireless client tunes itself to a specific channel, and solicits beacon

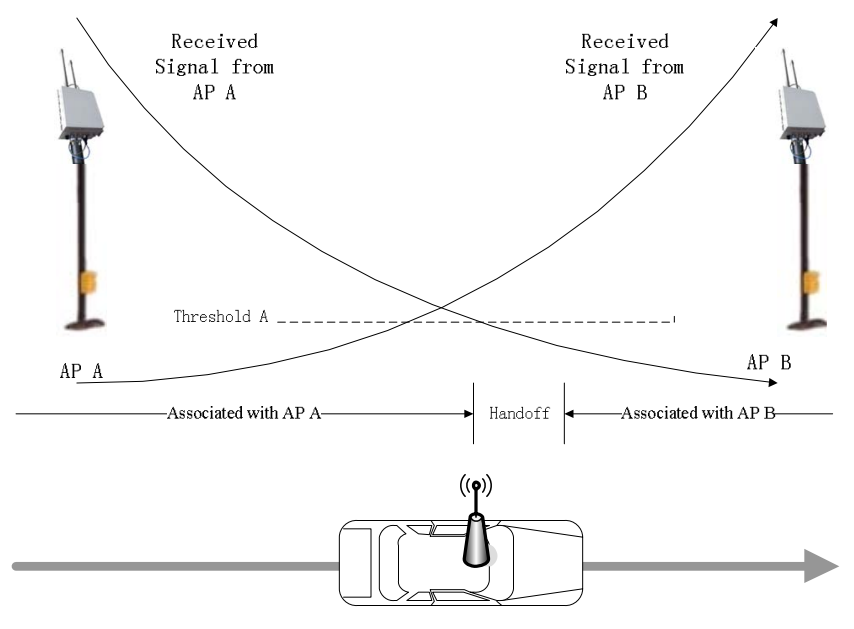

Figure 2. shows an ideal case for the L2 handoff process. In this scenario, a mobile client was set in a vehicle. While the car moves away from its current associated AP A to AP B, the received signal strength from AP A will go down gradually. If it is lower than a given threshold, one handoff will be triggered. By scanning channels, if the mobile client finds that AP B has stronger signal strength than AP A, it will set AP B as its new associated AP. messages using PROBE REQUEST frames. The client then waits for responses on each channel. For the latter, the wireless client tunes itself to a specific channel, and stays on that channel for up to $100 \mathrm{~ms}$ to receive all periodic beacon frames of all incumbent APs in that channel.

As soon as a station chooses the most suitable AP from its candidate AP list, it will try to authenticate/associate with that AP. If it is successful, this station will set this AP as its new associated AP. For enforcing all APs update their forwarding table respectively to have the right network path for this station, the associated AP will broadcast the MAC address of this STA by link layer (L2) Update frames, whose format is defined in Inter-Access Point Protocol(IAPP [4]), among the whole network immediately. After the above handoff process, a station can transmit and receive data through its new associated AP. Fig. 2 shows an ideal case for the L2 handoff process.

The pioneering WLAN measurement work can be traced at least back to 1996 [5].Since then, a progression of wireless network measurement efforts has provided insight into the network performance estimation and behavior analysis. At the beginning, just the low level channel behavior (such as bit error rate) in pairs of in-building wireless STAs was estimated [6]. Then by collecting network statistics data using Simple Network Management Protocol (SNMP), system traces and Ethernet sniffer etc, larger environments such as university campuses can be observed indirectly and the higher layer behavior, such as traffic load, characteristics, and user mobility can be measured [7-8]. In the last 3-4 years, several specific testbeds have been built up to monitor WLAN directly, which can cover a building-scale production 802.11 network (such as UCSB Meshnet [9] and UCSD Jigsaw [10], which include 25 and 150 monitor nodes respectively), and even overlay submetropolitan area scale networks (such as MIT Roofnet [11]).

However the research in the above test beds is mainly focused on stationary wireless performance, so far few results have been published for mobile environments. In [12-13], channel simulators have been used to observe the performance of $802.11 \mathrm{~b}$ WLAN in an emulated mobile channel. The authors of [14] measured the impact of slow user motion by a toy locomotive along a 5 meter long track. The maximum tested speed is 1 meter per second. They found that the modulation type, the maximum retransmission number, the experimental setting, and even the quality of power supply may have more important influences than the motion speed on the wireless performance. Both [15] and [16] measured the mobile performance of UDP and TCP traffic under different velocities, while [16] employed two access points and extended the coverage of its test bed by interconnecting them using an Ethernet cable.

Overall our measurement work is the first major effort in wireless mobile network measurement in terms of scale, methodology, and analysis. Our work outlines approaches for new methodological challenges due to large scale railway multi-hop networks. Our multi-layer packet analyzer can trace the network behaviors among multiple monitors and multiple layers simultaneously. 


\section{DATA COLLECTION AND MEASUREMENT METHODOLOGY}

Ethereal/Wireshark [17] and AiroPeek [18] are used to measure the network performance from wired and wireless sides of the monitored network respectively. Both tools can record packets with a $1 \mu$ s resolution timestamp. AiroPeek also can record the radio information of captured packets, such as wireless frequency, received power, etc. In mobility performance tests, GPS devices are used to track the location and velocity of the mobile clients.

To provide a global view of the entire performance of the monitored network, based on the open source projects, a offline multi-layer packet analyzer has been developed, which can trace the network behaviors among multiple monitors, multiple layer simultaneously. To analyze multiple packet traces from a global view, we needed to combine the contents of all traces together. For each channel, a synchronized trace is established to record all transmitted packets. To filter duplicates packets, the minimum required synchronization accuracy need be within a Distributed Interframe Space (DIFS) range. For 802.11a, DIFS is $34 \mu \mathrm{s}$, while for $802.11 \mathrm{~b}$ DIFS is equal to $50 \mu \mathrm{s}$.

\section{A. Measurement challenges}

Comparing with previous work, applying passive measurement approaches in large-scale multi-hop multichannel railway networks has to face the following new challenges:

(1) Traditionally in an indoor environment, propagation delay can be simply omitted due to the small distances. Therefore a given packet, which is received by multiple monitors, can be treated as a reference packet, which is a simultaneous event for all potential interactions. In a large-scale outdoor environment, the time synchronization among multiple monitor's packet traces needs to consider the impact of the propagation latency. Otherwise, the accumulated measurement error caused by propagation latency may exceed the required synchronization accuracy. It will be up to $40 \mu$ s for the end to end communication in our UNL-FRA test bed.

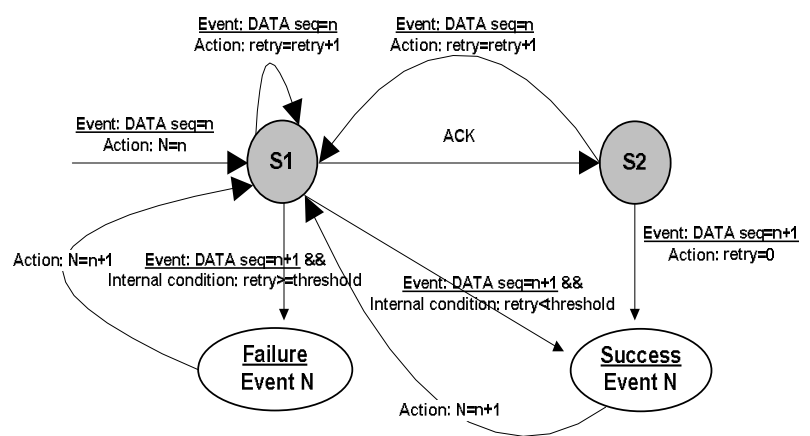

Figure 3. A FSM for correcting the status of captured DATA frames by using its context information. Each DATA packet carries a 12-bit sequence number. If following a DATA packet with sequence number $n$ is an ACK packet and a DATA packet with sequence number $n+1$, we can judge this DATA packet has been successfully transmitted in the actual traffic. Otherwise if following it is a DATA packet still with sequence number $n$, the previous transmission should be failed.
(2) In the case where both the antenna gain and height of a monitor are less than those of the outdoor AP towers, multiple monitors need to be employed simultaneously to record the network behaviors in a single channel. Each one records only partial behaviors in that channel. Therefore the packet traces collected from multiple monitors need to be merged together to establish a synchronized trace representing all transmitted packets in that channel from a global perspective.

(3) Due to propagation differences, the status of a packet in monitor traces may be different to the actual status of that of the monitored traffic. For example, a packet captured without any bit error in a monitor trace may be transmitted unsuccessfully in the actual traffic.

\section{B. Our measurement approaches}

To address the above challenges, our measurement solution, similar to [19] and [20], exploits the broadcast nature of wireless. The basic idea is close to [20]: a wireless channel essentially is a broadcast medium, so a transmitted packet can be recorded by multiple in-range receivers and can be used as a simultaneous event to synchronize them.

A universal procedure for merging multiple packet traces is designed, which includes the following five steps:

(1) Identify the pairs of reference packets from multiple packet traces;

(2) By using reference packets as simultaneous events, synchronize and merge multiple packet traces together, and remove duplicate packets.

(3) Compensate the propagation latency by the GPS information of the transmitter and receiver.

(4) Use packet traces from partial monitors, which periodically scan through multiple channels, as intermediate packet traces to synchronize packet traces across-hop across-channel.

(5) Use a set of finite state machines (FSM), which use one captured packet's context to estimate its actual status and each FSM is designed to correct the status of a specific packet type.

In our approach, beacons generated by APs are preferably chosen as reference packets, since they carry a 64-bit microsecond granularity timestamp and usually are transmitted with maximum power. Fig.3 uses a simplified FSM as an example to show how to correct the status of captured DATA frames.

\section{Mobile environment measurement approaches}

To measure the mobility performance, mobile stations and monitors are set up on a HyRail vehicle, which shuttled along the railway between AP 1 and AP 8 several times under different velocities. The monitors are set to capture 3 nonoverlapping $802.11 \mathrm{~b}$ channels $(\mathrm{CH} \quad 1,6$ and 11) simultaneously. To minimize the influence of the inconsistencies from the monitor's system clock, specific software has been developed to synchronize the system clock of all monitors at the beginning of each field experiments. During the mobility experiment, the Ethernet ports of the monitors are interconnected by a hub. Wired broadcast packets, 
which are transmitted periodically by one of the monitors, are used as reference packets to synchronize the system clock of the monitors.

\section{MOBILITY PERFORMANCE MEASUREMENT RESUlTS}

Using the above measurement approaches, we can maintain clock synchronization among wireless monitors with microsecond accuracy during the mobility performance tests. The measurement results for the velocity impact of BER conclude that BER performances under different velocities are close. When the service coverage of the APs is fixed, a mobile STA with higher velocity usually has a lower average throughput than a slower one. This is not due to signal degradations, but because it spends a larger fraction of time on scanning channels.

In this section, we present the measurement results of the QoS of the VoIP application in the mobile environment. In general, there are four main QoS characteristics: bandwidth, packet loss, latency and availability, which is the availability ratio of network services during a certain amount of time. The human perceived voice communication QoS can be expressed by a single characteristic: MOS (Mean Opinion Score), which relates directly to end user experience and expectations [21]. For good voice quality the corresponding MOS should be between 4.0 and 5.0, acceptable (in the range of 3.1 to 4.0 ), and poor (less than 3.1).

The transmission rating factor $\mathrm{R}$, which can be mapped one-to-one to an estimated MOS, is calculated by the following equation [22]:

$$
R=R_{0}-I_{s}-I_{d}-I_{e-e f f}
$$

Where $R_{0}$ represent subjective quality impairments, $I_{s}$ represents the subjective quality impairments due to loudness, side tone, and quantization distortion. These two parameters are not associated with the network transmission. $I_{d}$ is related to the network delay, which represents subjective quality impairments due to talker echo, listener echo, and end to end delay, and $I_{e-e f f}$ represents subjective quality impairments due to packet loss.

An actual multiple layer measurement analysis example of the VoIP mobility communication process is showed in Fig.4. The data was collected while a STA shuttled between AP A and AP B, while channel 1 and channel 6 were assigned to AP $A$ and $B$ to access STAs respectively.

It is clear that even in such short experiment duration (only 120 seconds), there are two severe performance attenuations due to the uncertain handoff latency. Not only QoS cannot be guaranteed, even the seamless service cannot be provided. In fact, the test bed is designed to provide overlapping coverage areas for all locations.

After filtering all handoff intervals, we can show the VoIP QoS under different network conditions in Fig.5. There is no QoS based forwarding mechanism in the current 802.11 implements so real-time traffic flows cannot be transmitted
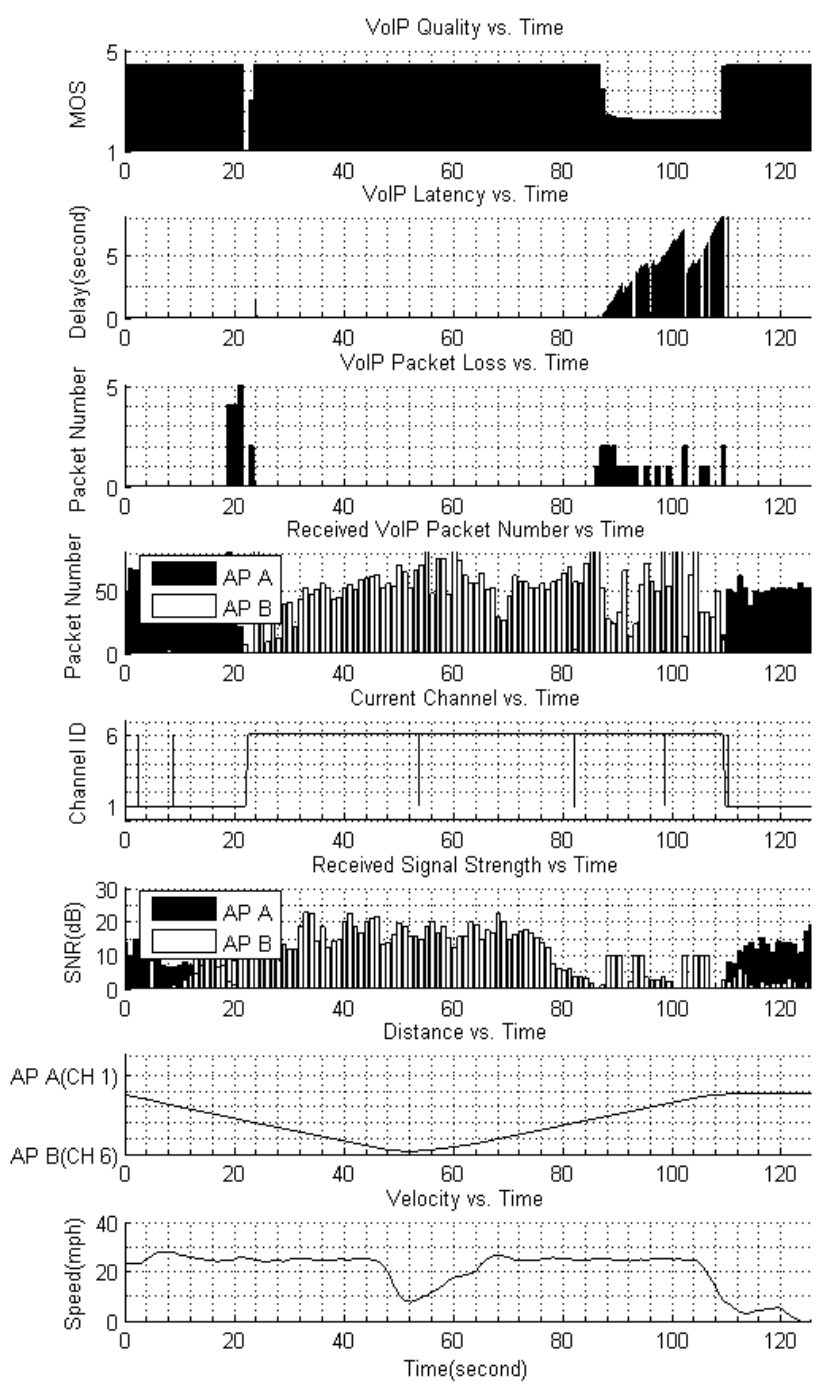

Figure 4. A multiple layer analysis example for the mobility performance. Our passive measurement approaches can provide a global view of the entire performance of the monitored network: from the physical layer (channel ID, RSSI, etc) to the real-time application performance.

with higher priority in the presence of background traffic. This results in much higher latency and severely impairs the quality of the voice communication.

\section{ANALYSIS OF Results}

The measurement results obtained for mobility performance shown in the previous sections aim to answer the central question investigated in this paper: what are the performance bottlenecks in large-scale mobile railway networks? Our measurement results show that although the current 802.11 implementations can provide much higher throughput for clients than that of $3 \mathrm{G}$ cellular technologies, they cannot guarantee QoS and may also have difficulties to provide seamless mobile service due to differences in the client device implementations. In the following, we will analyze the 


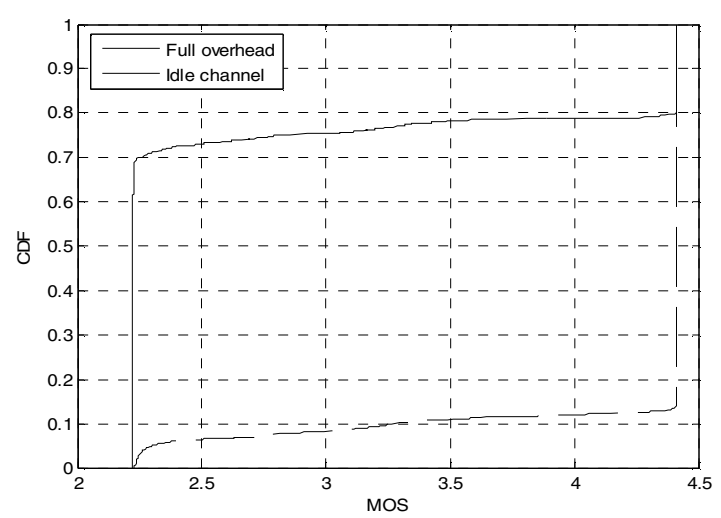

Figure 5 shows the VoIP QoS under different network overheads. After filtering all handoff intervals, the influences of packet loss can be omitted. The voice communication quality is mainly impaired by packet latency. Because without QoS based forwarding mechanism, the real-time flows cannot be transmitted with higher priority in the presence of background traffic, higher background traffic will bring higher packet latency.

optimization implications for enhancing the mobility performance in current 802.11 implementations.

An adaptive handoff trigger configuration algorithm should be proposed to provide the means for a trade-off between roaming response latency and maximum throughput, because while scanning channels, a STA has to interrupt its current data transmission. Lower roaming response latencies would result in a higher frequency of channel scans and thus a lower throughput for the STA. Therefore the handoff trigger conditions, such as a given signal strength threshold or a given retried packet number threshold, need to be adaptive to the channel status.

Also the information exchange between APs and STAs should be standardized. Usually handoffs are initiated by STAs. Without notifications from APs, a STA has to trigger scanning channel and choose its next AP based solely to its local knowledge. So from the operational network perspective, it is very difficult to predict a mobile wireless client's actions. By using IEEE 802.11f Inter Access Point Protocol (IAPP), APs can exchange the context information about STAs, however so far there is no standard designed for the information exchange between APs and STAs. Proprietary protocol extensions do not provide an adequate solution to this problem.

\section{CONCLUSIONS}

We present our quantitative measurements of a mobile wireless Wi-Fi network work based on our 3.5 mile Wi-Fi test bed on BNSF track in Crete Nebraska. Our measurement approaches are passive and do not introduce traffic overhead or affect performance. In order to address the measurement challenges posed by both network scalability and mobility, a unique measurement procedure is proposed to synchronize the system clock of distributed monitoring systems, merge multiple packet traces across-hop across-channel together, correct captured packets' status and analyze the performance of the monitored network by a global perspective. Our measurement results show that in an outdoor 802.11 railway environment, the current technology can provide much higher throughput for clients than 3G technologies. However they cannot guarantee QoS, or a seamless mobile service due to uncertain handoff latencies and no lack of prioritized stream-based forwarding mechanisms.

\section{ACKNOWLEDGMENT}

This work was funded by the Federal Railroad Administration (FRA) and supported by the Union Pacific Railroad and the BNSF Railway.

\section{REFERENCES}

[1] Standard, ANSI-IEEE 802.11. Wireless LAN Medium Access Control (MAC) and Physical Layer (PHY) Specifications 802.11 1999.

[2] M. V. Clark, K. K. Leung, B. McNair, and Z. Kostic. Outdoor IEEE 802.11 cellular networks: Radio link performance. IEEE ICC 2002.

[3] M. Hempel, H. Sharif, T. Zhou, and P. Mahasukhon. A Wireless Test Bed for Mobile 802.11 and Beyond. IEEE International Wireless Communications \& Mobile Computing Conference(WCNM) 2006.

[4] IEEE. Recommended Practice for Multi-Vendor Access Point Interoperability via an Inter-Access Point Protocol across Distribution Systems Supporting IEEE 802.11 Operation, IEEE Std P802.11F/D6

[5] D. Eckardt, P.Steenkiste. Measurement and Analysis of the Error Characteristics of an In-Building Wireless Network. ACM SIGCOMM 1996.

[6] Diane Tang, Mary Baker. Analysis of a local-area wireless network. ACM MOBICOM 2000.

[7] D. Kotz, K.Essien. Analysis of a Campus-wide Wireless Network. ACM MOBICOM 2002.

[8] T. Henderson, D. Kotz, and I. Abyzov. The Changing Usage of a Mature Campus-wide Wireless Network. ACM MOBICOM 2004.

[9] http://moment.cs.ucsb.edu/meshnet

[10] Yu-Chung Cheng, John Bellardo, Péter Benk, Alex C. Snoeren, Geoffrey M. Voelker, Stefan Savage. Jigsaw: solving the puzzle of enterprise 802.11 analysis. ACM SIGCOMM 2006.

[11] D. Aguayo, J. Bicket, S. Biswa, G. Judd, and R. Morris. Linklevel measurements from an 802.11b mesh network. ACM SIGCOMM 2005.

[12] C. Steger, P. Radosavljevic, and J. P. Frantz. Performance of IEEE 802.11b Wireless LAN in an Emulated Mobile Channel. IEEE Vehicular Technology Conference(VTC) 2003.

[13] C. Steger, P. Radosavljevic, and P. Frantz. $802.11 \mathrm{~b}$ operating in a mobile channel: Performance and challenges. CDC 2003.

[14] Christian Hoene, A. Günther, and Adam Wolisz. Measuring the impact of slow user motion on packet loss and delay over IEEE $802.11 \mathrm{~b}$ wireless links. Workshop on Wireless Local Networks (WLN) 2003.

[15] J.Ott, D.Kutscher. Drive-thru internet: IEEE $802.11 \mathrm{~b}$ for automobile users. IEEE INFOCOM 2004.

[16] J. Ott and D. Kutscher. A Disconnection-Tolerant Transport for Drivethru Internet Environments. IEEE INFOCOM 2005

[17] http://www.wireshark.org/.

[18] http://www.wildpackets.com/products/.

[19] Ratul Mahajan, Maya Rodrig, David Wetherall, John Zahorjan. Analyzing the MAC-level behavior of wireless networks in the wild. ACM SIGCOMM 2006.

[20] J. Elson, L. Girod, and D. Estrin. Fine-Grained Network Time Synchronization using Reference Broadcasts. the Fifth Symposium on Operating Systems Design and Implementation(OSDI) 2002.

[21] ITU-T Recommendation P.800, Methods for subjective determination of transmission quality 1996

[22] VoIP Evaluation for MBWA (Rev.1) http://grouper.ieee.org/groups/802//20/Contribs/C802.20-05-36r1.doc. 